Commun: Communication et organisation

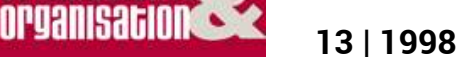

Management par projet et logiques

communicationnelles

\title{
Paradoxes et devenirs de la presse d'entreprise française
}

Pascal Lardellier

(2) OpenEdition

Journals

Édition électronique

URL : http://journals.openedition.org/communicationorganisation/2046

DOI : 10.4000/communicationorganisation.2046

ISSN : 1775-3546

Éditeur

Presses universitaires de Bordeaux

Édition imprimée

Date de publication : 1 mai 1998

ISSN : 1168-5549

Référence électronique

Pascal Lardellier, «Paradoxes et devenirs de la presse d'entreprise française », Communication et organisation [En ligne], 13 | 1998, mis en ligne le 26 mars 2012, consulté le 19 avril 2019. URL : http:// journals.openedition.org/communicationorganisation/2046; DOI : 10.4000/

communicationorganisation. 2046

Ce document a été généré automatiquement le 19 avril 2019

(c) Presses universitaires de Bordeaux 


\title{
Paradoxes et devenirs de la presse d'entreprise française
}

\author{
Pascal Lardellier
}

\section{NOTE DE L'AUTEUR}

L'étude présentée ici se fonde sur une enquête réalisée par l'auteur auprès d'une soixantaine d'entreprises de Nice, Cannes et Monaco possédant un journal interne, ainsi que sur quelques années de conseil et formation en journalisme d'entreprise. Cet article développe et " problématise » des recherches antérieures de l'auteur. D'abord, certains des chapitres de l'ouvrage Le journal d'entreprise, les ficelles du métier, éditions d'Organisation, Paris, janvier 1998 ; ensuite, quelques-uns des éléments de l'article paru dans Médias Pouvoirs en décembre 1996, intitulé « Le journal d'entreprise, mirage ou miroir ? Une analyse « sismographique de la presse d'entreprise française »

\section{Un malentendu persistant est lié à l'image et aux "finalités idéologiques » de la presse d'entreprise}

1 Les journaux d'entreprise occupent une place à part au sein des vecteurs et des politiques de communication interne. Plus de deux fois centenaires, sédentarisées, contre vents et marées, dans maintes organisations, ces publications ont toutefois revêtu pour la plupart d'entre elles pendant des décennies - et jusqu'à très récemment - un caractère "artisanal », qui les affranchissait de tout professionnalisme. Pourquoi ? Simplement parce que sous couvert de collégialité (et de l'indigence des moyens qui lui étaient alloués), la réalisation de cette publication était laissée à la libre initiative d'employés bénévoles, qui travaillaient avec pour seul viatique leur temps libre et leur bonne volonté, indispensables pour « faire vivre le journal interne ».

Cet amateurisme manifeste n'affectait pas seulement l'apparence du journal d'entreprise. Car jusqu'au milieu des années 80 , qu'en était-il, en vérité, du projet véritable de nombre 
de titres de la presse interne? À vrai dire, on entrait là en terra incognita. La seule jubilation qu'éprouvaient ces employés volontaires à se glisser, de temps à autre, dans la peau de journalistes exemptait de tout recul critique sur le contenu, de toute réflexion véritable sur les finalités de ce journal interne. Ce qui n'empêchait pas les conflits de pouvoir en son sein, et autour de lui.

Notre jugement est-il pessimiste, voire erroné ? Voire... En tout cas, nous avons pu souvent vérifier « sur le terrain » ces mauvais travers, difficilement justiciables, eu égard à la passion réelle de « l'équipe rédactionnelle » à animer et à pérenniser « leur journal », coûte que coûte.

Bien sûr, les journaux rutilants des grandes entreprises ${ }^{1}$, fréquemment réalisés "en externe " par des agences spécialisées ou par des journalistes professionnels constituent une façade, qui ne doit ne pas masquer ce constat: des objectifs mal définis, des moyens (et une périodicité) fluctuants, tels ont longtemps été les traits marquants d'une presse d'entreprise française, qui en dépit de son institutionnalisation, affiche encore à certains égards des imperfections criardes.

5 Mais concernant la presse d'entreprise, et alors que le cap symbolique de l'an 2000 se profile, le temps est-il toujours à cet amateurisme de bon aloi, qui affecte encore nombre de publications? Il semble que l'époque soit au contraire à une redéfinition radicale des missions, des modalités de réalisation, de l'apparence, et - numérisation oblige - du support même de ce journal d'entreprise. Accèdera-t-il pour autant au professionnalisme et, surtout, à la légitimité qui lui font encore parfois défaut ? Telle est l'unes des questions que pose la presse d'entreprise, quand on souhaite en dresser un bilan critique, à l'aube du XXI siècle.

6 En dépit de certaines faiblesses, et des critiques dont il est régulièrement l'objet, le journal interne possèderait-il un "supplément d'âme ", garant de son attrait, de son succès, et de sa pérennité ? Il est bien plus attractif, en tout cas, que la plupart des autres vecteurs de communication interne, neutres voire mornes.

7 Mais en règle générale, il est abordé (et "pratiqué ») de manière trop passionnelle, laissant peu d'employés insensibles. Et pour cause : dans l'idéal, tous peuvent y participer.

Du reste, une équation se vérifie fréquemment « en interne » : ceux qui ne décrient pas le journal cherchent à se l'approprier. Espace d'expression, d'information et de savoir, il est indéniablement porteur de pouvoir. Tour à tour objet d'un enthousiasme symptomatique et de critiques teintées de procès d'intention, la presse d'entreprise se trouve souvent prise dans des jeux d'influence et de pouvoir qui la dépassent. Ceux-ci la placent au centre de conflits symboliques opposant le patronat, l'encadrement, le comité d'entreprise et le personnel de « la base ». Alors, la collégialité et l'indépendance qui sont censés fonder le journal (dans l'absolu...), restent à trouver, et à prouver. Dans nombre d'entreprises, le journal interne est de même tiraillé entre les tentatives « d'inféodation » (en tout cas de contrôle) de la direction de la communication, et de celle des ressources humaines. Mais le personnel, répétons-le, n'est jamais indifférent au journal interne. Il existe, d'ailleurs, une ligne de partage symbolique dans les organisations, entre ceux qui participent et ceux qui ne veulent pas participer pas à ce journal d'entreprise, et surtout entre ceux qui le lisent, et ceux qui ne le lisent pas.

9 Enfin et surtout, il lui arrive d'être dénoncé par les syndicats, comme étant le cheval de Troie des stratégies participatives du patronat dans l'entreprise ou l'organisme. On rencontre là l'un des griefs les plus fréquents concernant la presse interne : sa «finalité 
idéologique ». En effet, le journal d'entreprise n'œuvre-t-il pas en fait, sous couvert d'information, à encourager le sentiment d'appartenance? Est-il l'instrument du patronat, se faisant par-là même la chambre d'écho de la culture d'entreprise, ou sert-il simplement l'information, la communication, et leur qualité «en interne »? Le fait est qu'il est difficile d'aborder ce problème sans être manichéen.

On voit souvent poindre, derrière les journaux d'entreprises et autres initiatives participatives, la dimension stratégique du plan de communication, dont la presse interne est l'une des clefs de voûte. On peut prêter en effet une part de machiavélisme à ce plan de communication interne: "le véritable objectif des politiques de communication de toute institution reste le renforcement pur et simple de leur féodalité, sous couvert d'ouverture. $»^{2}$ Bernard Floris, entre autres, a entrepris une critique radicale de la " communication managériale . $^{3}$

11 Force est de reconnaître que l'apparition de la communication d'entreprise est datée, et que l'institutionnalisation de la fonction et de la profession répondait au moins autant à un besoin hypothétique de communication au sein des organisations, qu'à une volonté de légitimation, de reconnaissance et de "modernisation symbolique», ainsi qu'à un recentrement de l'image de l'entreprise. La communication, valeur-refuge de la postmodernité, utopie et idéologie déclarées des années 80 , ne pouvait pas ignorer l'entreprise. Elle n'est pas venue naturellement aux organisations, mais son lit y a été fait alors que les instigateurs de cette union - partis prenants d'autant plus motivés qu'ils vivaient de ce genre de rapprochements - savaient qu'il allait s'agir d'une mariage d'intérêts, dont l'entreprise sortirait assurément gagnante, en tant que structure managériale.

12 Il serait d'ailleurs réducteur de considérer que seul le monde de l'entreprise, représenté par le patronat, et des bataillons de cadres cyniques, a œuvré à institutionnaliser la communication en tant que valeur et pratique stratégique. De nombreux indices nous confirment que dès le début des années 80 , la communication, paradigme triomphant, s'est imposée socialement et politiquement, comme système de valeurs et utopie (il faut bien employer le mot, à l'instar de Philippe Breton). L'apparition du Ministère de la communication, incarné par l'emblématique Jack Lang, ainsi que la Journée d'expression instituée dans les entreprises par la loi Auroux (datée de 1982) sont explicites, entre autres, de cette volonté politique autant qu'entrepreneuriale de légitimer l'émergence de la communication.

13 La communication d'entreprise s'appuie sur un éventail d'outils, de pratiques, et indéniablement, elle poursuit un but, œuvrant à atteindre des finalités managériales. Pour autant, la position est à notre sens extrême, qui consiste à opter pour une approche uniquement «balistique ", réduisant toute action en communication interne à un rapport tireur/cible, à une opération raisonnée manigancée par les dominants, directions et encadrements, «tendant à transformer les dominés en acteurs de leur domination », sous couvert de logique participative.

14 Une réflexion dialectique nous demande d'écouter tous les protagonistes. Face aux positions critiques vis-à-vis de la communication interne, qu'en est-il, par contre, du discours des tenants de celle-ci? En «interne ", l'information et la communication « doivent être conçues et envisagées comme des fonctions essentielles du management car elles contribuent fortement, à une époque de mutations importantes, à l'émergence d'une dynamique nouvelle de l'entreprise $»^{4}$ On se souvient, de même, du constat énoncé par Peters et Waterman dans leur best-seller Le Prix de l'excellence, et selon lequel c'est 
l'attention portée au personnel, plus que les conditions de travail, qui a un impact véritable sur le rendement. Ce que Mayo et Maslow avaient d'ailleurs prouvé dès les années 20 et 30. Et il va sans dire que cette attention passe au premier chef par les possibilités offertes aux employés de s'exprimer et de communiquer.

Soit. Il est difficile de trancher, et notre propos n'est pas de prendre position. Nous nous situerions d'ailleurs dans la zone médiane, celle qui en appelle à des pratiques de communication internes fondées non sur le martèlement subliminal des valeurs d'entreprise, non sur la répétition incantatoire de quelques leitmotivs, non sur la hiérarchisation de l'accès à l'information, ou la rétention de celle-ci, mais au contraire sur des valeurs, telles que la transparence, un accès égal à la parole et aux messages, l'écoute et le respect, (l'une et l'autre sous-entendant une " expression libre », et donc pas seulement cantonnée à l'injonction et à la revendication...).

Mais nous avons conscience de la difficulté de mise en œuvre, ainsi que sur la faiblesse relative de cette pétition de principe, sachant que les valeurs " en interne", si l'on veut qu'elles soient respectées, induisent souvent des chartes, qui elles-mêmes, prêtent à critiques... ${ }^{5}$

17 Sachant surtout, qu'à certains égards, la circulation d'information est un processus naturel, inhérent à l'organisation et à son fonctionnement. ${ }^{6}$ Il conviendrait alors de rationaliser et « d'humaniser » ces flux d'information.

18 Est-il d'ailleurs vain ou candide d'évoquer ici le respect dû aux personnes, et qu'une information complète honore? Montaigne affirmait déjà au XVIe siècle: "Je vois que chacun se mutine si on lui cache le fond des affaires auxquelles on l'emploie, et si on lui dérobe quelque arrière-pensée ». Cette sentence trouve une cruciale actualité de nos jours, dans maintes organisations. À la décharge de la communication d'entreprise, qui peut « s'épanouir « (le mot est certes connoté !) dans un contexte professionnel, travailler sereinement alors qu'il devine que des choses lui sont systématiquement cachées, et que les réalités sont accommodées? Or, il n'est pas sûr que ce respect soit encore présent, quand le personnel est obligé de compléter par le «canal informel «la parcimonie des informations "officielles "qu'il reçoit. C'est en ce sens que les partisans de la communication interne affirment que celle-ci n'est pas une dépense, mais un investissement pour l'entreprise. Elle coûte moins que peut coûter son absence. Et d'évoquer, en les chiffrant, les effets pervers dûs à l'absence de communication : rumeurs, désinformation, démotivation, absentéisme...?

19 Tout ne serait alors qu'affaire de rendement et de rentabilité ? À quitter un instant le pragmatisme et le cynisme, même, on les voit revenir au galop.

20 Sans vouloir épiloguer, ni renvoyer dos à dos deux écoles, nous ne pouvons qu'affirmer avec réalisme qu'il y a une dimension idéologique forte à affirmer que la communication d'entreprise est un investissement, comme à la critiquer, en tant que pratique managériale. Le débat n'est pas tranché, puisqu'il est finalement politique.

21 Et l'essentiel est sans doute d'avoir conscience des enjeux de ces discours, quand des initiatives telles qu'un journal interne, précisément, se mettent en place « en interne »; connaître et maitriser les lignes de force de ces positions antagonistes permet finalement de gagner du temps, tout en manœuvrant sans passion ni cynisme excessifs, en toute rigueur. 


\section{La question délicate des missions de la presse d'entreprise}

Mais quelles missions remplit en fait la presse d'entreprise? Quelques grandes fonctions lui sont en règle générale assignées, qui concernent autant l'expression, l'information que la communication : d'abord, il informe le personnel sur les grandes évolutions de la vie de l'entreprise, de ses salariés et de son milieu d'activités, sur les mutations de son environnement économique, concurrentiel et social. En période de crise, il doit aussi répondre aux grandes questions du personnel, afin d'être un outil efficace contre la rumeur, la désinformation. Il y a là un besoin, que ni les notes de service, ni les tableaux d'affichage... ne peuvent combler. Tel est alors le créneau du journal. Et gageons qu'une information peut être institutionnelle, en gardant une validité et une crédibilité.

23 Mais ce journal doit aussi permettre de fédérer les employés de l'organisation, en favorisant leur esprit d'appartenance à l'entreprise. Avoué ou non, cet objectif est l'un des points de fuite de toute action en communication interne, en même temps qu'il constitue la pierre d'achoppement idéologique que nous avons évoqué précédemment.

Le journal interne doit aussi encourager les contacts, les rencontres, les collaborations, permettant à des représentants de toutes les catégories d'échanger et de communiquer avec une certaine liberté.

Ensuite, il doit mettre en valeur les initiatives et les compétences, autant professionnelles que personnelles (faire découvrir, par exemple, que l'on travaillait sans le savoir à côté d'une peintre ou d'un marathonien...).

L'une des questions de l'enquête que nous avons menée en 1996 auprès de soixante responsables de journaux d'entreprises de la région PACA avait pour but de savoir quelles étaient les fonctions idéales remplies par ce journal, à leurs yeux. Les réponses furent explicites, et quasi-unanimes : " décloisonner ", d'abord. Nous sommes là dans le champ de la communication et de l'expression, avant même - ou par delà - l'information. Donc, et selon le postulat célèbre de Watzlawick, « la relation prime sur le contenu ». Ou en tout cas l'enrichit.

Il est de même un lien précieux entre des sites délocalisés, un trait d'union entre des catégories de personnels qui ne se croisent que rarement, du fait d'horaires différents, de formations ou de congés longs, éloignant certains employés de l'entreprise... Mais cette fonction de médiation, il peut aussi la remplir auprès des anciens employés (en retraite) ou des partenaires réguliers.

C'est enfin souvent à lui qu'incombe d'être le porte-étendard de la culture d'entreprise, et de la mémoire de l'entreprise auprès du personnel. A certains égards, il est le visage de l'organisation, tendu autant à ses membres qu'à ses interlocuteurs extérieurs. Ceci va encore dans le sens de cette reconnaissance et de ce sentiment d'appartenance, que l'on cherche par son biais à développer.

On peut le constater, le journal interne se voit assigner des fonctions disparates. Ceci renvoie dos à dos l'amateurisme, l'empirisme et la spontanéité sous l'égide desquels il est souvent réalisé.

En regard de ces objectifs, le journal s'adresse à des publics hétérogènes, et dans l'idéal, chacun devrait y trouver des réponses à ses attentes et à ses préoccupations. Il doit plaire, 
mais à qui ? Là est le problème: autant à ses lecteurs, dans toute leur diversité hiérarchique, qu'aux dirigeants qui l'autorisent et le financent.

On comprend la complexité de rédaction et de réalisation d'un journal pensé, s'inscrivant dans un projet, et qui se veuille vraiment l'expression de l'entreprise et de tous ses employés. On imagine surtout l'abnégation et la diplomatie dont doivent faire preuve ses principaux acteurs. Loin d'être le passe-temps de quelques stagiaires en communication, ou la marotte de cadres en préretraite, il trouve là une dimension participative, bien sûr, mais aussi humaine, et finalement humaniste, aspect sur lequel nous reviendrons au moment de conclure.

\section{La presse d'entreprise se caractérise par une hétérogénéité confondante}

32 Retour circulaire au titre de cet article. Nous évoquions les paradoxes qui semblent inhérents à la presse d'entreprise. Ce domaine est un genre qui désespère presque toute tentative de catégorisation, tant les styles, les missions, les tons et les sommaires, les tirages, les formations et niveaux de compétence, les expériences, les publics foisonnent, pléthoriques à l'envi. Cette extrême diversité ne manque pas de receler quelques paradoxes, qui doivent être énoncés.

33 Le journal d'entreprise est pluriel par nature; et en tant que vecteur de communication interne, il sort sans doute plus affaibli qu'enrichi de cette diversité. Car ce que l'on a tendance à retenir, en règle générale, ce sont les défauts, l'amateurisme, les maladresses, les manquements aux missions. Qu'on juge de l'hétérogénéité de la presse d'entreprise française par cette rapide revue de détail.

Il faut d'abord rappeler que le journalisme d'entreprise est un phénomène essentiellement parisien. Si plus d'une trentaine de départements français ne sont même pas représentés à l'UJJEF, ${ }^{7}$ la moitié de ses membres sont domiciliés à Paris et dans les Hauts-de-Seine. En 1995, d'ailleurs, 69 \% des organisations adhérentes étaient encore concentrées en Ile de France, ce qui trahit une disproportion étonnante. ${ }^{8}$ La presse d'entreprise se caractérise donc par une répartition géographique très inégale de ses titres en France (si tant est que l'adhésion à l'UJJEF soit représentative de la « distribution » des journaux sur le territoire).

Ce caractère disparate concerne aussi les tirages ; ainsi, alors que certains journaux sont diffusés à des milliers d'exemplaires, d'autres, au contraire, sont édités à des tirages quasi-confidentiels.

Parlant de l'hétérogénéité de cette presse, il faut aussi rappeler que tous les secteurs d'activité ne sont pas «logés à la même enseigne». Des constatations qui déroutent souvent sont d'ailleurs faites sur ce point. Bien sûr, le domaine des services, les grandes administrations, le tertiaire et les industries de pointe sont très bien représentés, et la plupart des organisations issues de ces milieux possèdent un journal interne. Il n'en va pas de même, par contre, pour l'industrie lourde, le bâtiment et le commerce. Car le nombre de salariés n'est en rien un indicateur fiable de l'existence d'un journal au sein de l'entreprise, comme on pourrait le penser. Il arrive qu'un organisme de Sécurité sociale comptant à peine cent agents édite une magnifique publication bimestrielle, alors que les grands noms de l'industrie du parfum des Alpes Maritimes, par exemple, qui comptent de trois cents à six cents employés, ne possèdent aucun journal interne, et avouent n'en 
avoir jamais eu... À quoi cela est-il dû ? Peut-être au fait qu'il reste des secteurs dans lesquels la communication ait encore à prouver son bien-fondé, son utilité et sa rentabilité. Cela dépend aussi, et surtout de la position de la Direction générale de l'organisation vis à vis de cette communication. À son égard, il est des structures ouvertes, et d'autres réfractaires.

Qu'en est-il du ton de ces journaux internes? Il trahit hélas fréquemment qu'il est bien difficile de rendre le journal attrayant et vivant. Trop souvent, le ton est banalement corporate, lénifiant et institutionnel, au mauvais sens du terme. Réalisé non pour que les salariés s'y reconnaissent, il est alors uniquement destiné à vanter les performances de l'entreprise, « l'excellence de nos produits » sur ceux des concurrents. Le ton peut même s'apparenter à de l'auto-publicité, voire à une propagande d'entreprise éhontée. Dans ces cas-là, pas lu, critiqué, raillé, le journal s'attire rapidement l'indifférence ou la mésestime. Comme variante, il peut adopter un jargon tellement technique qu'il n'intéresse personne, et rebute tout le monde. Ces erreurs de fond découlent d'une méprise, ou pire, d'un mépris total de la prise en compte de ce qu'attendent les lecteurs, voire d'une falsification des missions du journal. Elles génèrent alors du ressentiment, dont la communication en tant que pratique, pâtit au premier chef.

Les sujets traités, à l'instar du ton qui les exprime, sont aussi très hétérogènes. De nature technique, institutionnelle, sociale, sectorielle, économique, les comités de rédaction ont bien du mal à trouver un sommaire qui soit à la fois cohérent et crédible.

Le meilleur des parti pris, pourtant, n'est-il pas de parler autant de la vie de l'entreprise que des préoccupations quotidiennes des salariés, afin que ceux-ci sentent dans ce journal un miroir à leur quotidien, un canal d'expression à leurs projets, à leurs inquiétudes, à leurs attentes ? Il faut aussi ouvrir un tant soit peu les sujets des articles à l'extérieur de l'entreprise, à son environnement économique et technique, mais aussi culturel, social et humain. Ceci permet de donner aux lecteurs une meilleure compréhension des ensembles dans lesquels l'organisation est insérée, d'en avoir une intelligence plus fine.

Au rang des disparités, la forme et l'apparence même du journal tendent aussi à le desservir. Dans les PME et dans nombre d'entreprises qui n'ont pas compris que le journal doit être professionnel ou ne pas être, c'est souvent une revue à l'aspect amateur, une publication « de patronage », qu'un véritable outil de communication interne. À l'inverse, il est professionnalisé à l'extrême dans certaines grandes entreprises, qui vont jusqu'à employer plusieurs journalistes diplômés pour «sortir» des publications de luxe, emblématiques autant de l'esprit maison que des moyens somptuaires investis dans «la communication ». (Quoique les journaux d'entreprise aient pâti au premier chef, depuis le début des années 90 , de la crise des budgets qui étaient encore récemment alloués à la «com. interne»; ou de la migration de ceux-ci vers des investissements plus « rentables », comme l'informatique par exemple.)

41 Enfin, les acteurs du journal ont souvent des profils extrêmement variés. Du contremaître qui a appris à écrire sur "le tas » au jeune cadre qui "rôde sa plume», de la personne venant enfin de se voir proposer une formation en "journalisme d'entreprise» au stagiaire qui valide son DESS «Communication des organisations » en s'acquittant de la réalisation du journal, et jusqu'au journaliste professionnel qui rewrite à ses heures des publications interne au style incertain, le moins que l'on puisse dire, c'est que les acteurs de la presse interne ne possèdent pas de profil-type. L'implication de chacun dans le comité de rédaction du journal étant de plus liée à des motivations différentes, il est 
souvent bien difficile de trouver des modes de collaboration conciliant tous les membres du comité de rédaction.

De multiples facteurs expliquent cette disparité générique, à chercher dans la structure et la culture propres à chaque entreprise. Enfin, il ne faut pas oublier que le degré de professionnalisme de la communication dans une organisation donnée, se retrouvera proportionnellement toujours dans le degré de professionnalisme du journal. Il n'y a là ni secret ni surprise : le cursus et la formation des rédacteurs, leur aptitude à appliquer une méthodologie journalistique se ressent dans la tonalité générale de la publication. Il faut alors oser l'affirmer, quitte à dénigrer un travail amateur qui touche vite ses limites : seule une (auto-)formation systématique et rigoureuse des rédacteurs des journaux internes aux techniques d'écriture mais aussi à la déontologie journalistiques permettra de diminuer les disparités de forme et de fond de la presse d'entreprise, et de fédérer sous une apparence vraiment professionnelle, un genre jusque là trop fugace, et de ce fait encore trop peu crédible.

Nous n'en appelons pas à un hypothétique nivellement des formes de la presse d'entreprise au profit d'un modèle standard qui la rendrait moins hétérogène, et par-là, plus attractive. Réaffirmons qu'elle est plurielle par nature, et qu'elle tire sans doute aussi sa richesse de cette diversité. C'est par contre un plus grand professionnalisme qui permettra à cette presse d'entreprise de gagner définitivement ses galons les plus convoités, la lisibilité, la crédibilité et la légitimité, celles-ci lui faisant encore parfois défaut.

\section{Les horizons numériques de la presse d'entreprise}

C'est souvent à tort que la presse d'entreprise est perçue comme étant d'apparition somme toute récente, ayant émergé il y a quelques décennies tout au plus. Apparus en fait dès le XVIII ${ }^{e}$ siècle en Angleterre, bien présents au XIX en Allemagne et aux ÉtatsUnis ${ }^{9}$, les journaux internes ont eu le temps d'expérimenter des contenus et des missions, des acteurs et des publics, un modèle, donc, ayant eu historiquement un «support papier $» .^{10}$

Cependant, si les mutations que traversent la presse interne sont autant liées à des évolutions managériales qu'à des restrictions budgétaires, elles sont aussi technologiques, les supports évoluant depuis le début des années 90, métamorphosés par la numérisation. Non seulement elle ne peut se soustraire aux bouleversements que traversent actuellement les domaines de l'information et de la communication, mais elle devrait même jouer un rôle précurseur, pionnier, au sein de cette révolution en marche. De même qu'elle peut œuvrer à vulgariser, et à démystifier les NTIC, en offrant de celles-ci un emploi raisonné et convivial dans les organisations.

Ceci tend à confirmer que le journal d'entreprise est un contenu et un projet, avant d'être un média fixe et immuable.

Mais concrètement, quelles améliorations les réseaux numériques apportent-ils à la presse d'entreprise? D'abord, c'est la communication de l'entreprise en général qui est redevable vis-à-vis de ces nouveaux outils d'une efficacité accrue, et qui concerne la rapidité des échanges, le stockage, la diffusion et l'utilisation des informations... Empruntant les voies de messageries internes aux entreprises tel Intranet ${ }^{11}$, les données de toutes natures circulent sans contraintes, et sont accessibles à tous les employés 
équipés d'ordinateurs (ce qui sous-entend parfois un certain niveau hiérarchique...). Ceux-ci, grâce à un code d'accès, peuvent consulter ces données, mais aussi se les approprier pour les exploiter, les « retravailler », les enrichir puis les rediffuser, à toutes fins utiles.

Grâce aux supports numériques, c'est d'abord le sommaire du journal qui peut désormais varier et évoluer dans le temps, en continu et sans limites. Le caractère immuable et rigide des journaux sur papier est désormais caduc. Ainsi, des informations sont ajoutées ou développées au fil des jours, d'autres enlevées quand elles sont obsolètes... Quant aux données vraiment importantes (chartes de qualité, archives, organigrammes, cahiers des charges techniques...), elles peuvent figurer en permanence dans le journal. Mais consultables seulement par ceux qui le désirent.

Les messageries révolutionnent aussi la périodicité classique de la presse d'entreprise. Grâce aux réseaux, les journaux ne suivent plus des rythmes extrêmement lents (un journal tous les deux, voire tous les trois mois). Mais au contraire, le responsable de la communication a désormais la possibilité d'envoyer des messages très fréquents.

Et surtout, une entreprise entière peut prendre connaissance d'un même message, dès la conception de celui-ci et en simultané, faisant fi des délais de fabrication et de diffusion inhérents au support papier.

$51 \mathrm{Au}$ sein du sommaire, les articles intéressant un lecteur peuvent être approfondis, grâce au principe de consultation de l'hypertexte (caractéristique du CD-Rom par exemple). Chaque lecteur compose donc son propre sommaire, «zappant » les textes dont il n'a pas besoin, et consacrant au contraire un intérêt accru à ceux qui concernent son domaine de spécialisation (étant entendu que tous les textes peuvent être imprimés, et même en couleur). Ainsi, un technicien pourra se familiariser de manière interactive avec les particularités d'une nouvelle machine. Et il pourra tout aussi bien copier sur son disque dur l'article complet, pour compléter son fichier de fiches techniques. D'autres employés, par contre, consulteront sur le journal interne l'organigramme, faisant connaissance avec les nouveaux arrivants, lisant de courtes interviews de ceux-ci; et découvrant ces nouveaux visages grâce à la présence de photographies couleur numérisées !

Ces lecteurs souhaitent-ils d'ailleurs envoyer un message de bienvenue - ou simplement de travail - à ces employés qui arrivent dans l'entreprise? Rien de plus facile, car les réseaux sont interactifs. Chacun dans l'entreprise possède une boîte aux lettres, qui peut recevoir des messages, et qu'il suffit de consulter régulièrement, à l'aide d'un code personnel.

Surtout, chaque service au sein d'une même entreprise (ou chaque site en cas d'entreprise délocalisée), a désormais la possibilité d'éditer sa propre page, ou sa rubrique spécifique. Celles-ci viennent s'insérer dans le vaste patchwork que constitue alors le journal, le responsable de celui-ci étant garant de la cohérence générale de l'ensemble. Le journal est à ce titre d'une grande souplesse, et d'une variété extrême.

D'ailleurs, chacun dans l'organisation peut devenir acteur de la publication, en éditant un article. Répondant ainsi à sa vocation de collégialité, le journal dispose soudain d'un comité de rédaction comptant potentiellement autant de membres que l'entreprise compte de salariés! Et comble du paradoxe, tous les salariés peuvent participer à son élaboration... sans se rencontrer.

Qu'en est-il du coût de ces nouveaux outils ? Indéniablement, les réseaux tel Intranet font réaliser de substantielles économies d'échelle, pour ne prendre que le domaine de la 
presse d'entreprise. Un journal qui a migré sur réseau permet de ne plus payer «que » le travail rédactionnel, en faisant l'économie de toute la partie "fabrication» (montage, photogravure, impression, façonnage, sans même évoquer le papier, les encres et les frais de diffusion). Le journal n'est plus un support spécifique; «virtuel», il ne fait qu'emprunter les lignes téléphoniques, pour apparaître sur les écrans des ordinateurs.

Certains affirmeront que la qualité graphique de la presse interne version papier gardera toujours une prééminence, au regard du caractère imparfait du journal numérique, seulement consultable sur écran. Ceci était vrai il y a quelques années encore, mais le sera de moins en moins, tant la qualité générale des ordinateurs s'est améliorée. Ainsi, les nouvelles générations de "machines» permettent un «rendu» quasi-parfait des couleurs; elles intègrent des photographies numériques à très haute définition. Et ce que n'autorisera jamais le journal papier, on peut désormais incorporer des animations au sein même des pages, au fur et à mesure qu'elles défilent : petites flèches tournant pour attirer l'attention, dessins et logos clignotant; et d'ores et déjà, animations-vidéo intégrées.... permettant de voir de visu celui que le journal a interviewé, par exemple, alors qu'il répond aux questions en souriant !

Ces transformations rapidement décrites amènent-elles à penser que la presse d'entreprise sera bientôt virtuelle? Au regard de toutes leurs possibilités, les messageries numériques incarnent-elles la presse d'entreprise de demain, qu'elles dématérialisent paradoxalement? Il convient quand on aborde l'avenir des médias de ne pas être simpliste. Car quoi qu'on en dise, la limite de ces nouveaux vecteurs tient à leur nature technique même. Qui pourrait passer seulement deux heures par jour à «feuilleter » des journaux et des magazines devant son ordinateur, sans éprouver beaucoup d'inconfort, et sans sérieusement s'abîmer les yeux à terme?

Il y a bien peu de chances pour que l'on lise bientôt son quotidien, des magazines ou des romans sur écran, contrairement à ce que certains " prophètes » annoncent hâtivement. ${ }^{12}$ Jusqu'à un certain point, les journaux internes pourront être lus sur écran. Mais si les textes sont intéressants, les lecteurs imprimeront les articles pour les relire confortablement, et surtout les garder... Et on reviendra ainsi circulairement au support papier.

De plus, ces contraintes techniques inhérentes à l'ordinateur induisant une fatigue, déjà oculaire, vont dans le sens d'un raccourcissement général de la longueur des textes. Les longs articles ne sont pas lus sur écran, il faut donc concentrer l'information, et offrir des modules de lecture de plus en plus réduits. Ceci n'est pas sans incidences sur les contenus. Il est donc plausible qu'à moyen terme, les sujets «au long cours» retrouvent des supports papier.

60 Certes, la tendance est actuellement à une irréversible migration des données, depuis le traditionnel papier, vers les médias numériques. Et de plus en plus d'entreprises optent pour cette solution radicale, qui consiste à supprimer purement et simplement le journal papier au profit de son concurrent numérique.

61 On peut cependant penser que la presse "classique » sur papier ne disparaitra pas complètement. Loin s'en faut. On se dirige davantage vers une complémentarité entre les deux médias. La famille du livre, dont la presse (d'entreprise) fait partie, n'a pas dit son dernier mot. Un journal se regarde, se garde, se collectionne, s'emmène "sous le bras ", se lit dans le bus ou au café, il se commente en groupe... autant de choses toutes simples que l'ordinateur aura du mal à offrir. 

reclassement induit par « la logique de l'usage $»^{13}$; en naîtra sans doute un genre hybride, qui reste en tout cas encore à inventer, et dont les formes restent à penser.

\section{Vers l'ingénieur en communication interne...}

Les bouleversements décrits ici, dont certains sont à venir, ne font que rendre plus diversifiées et plus cruciales les multiples tâches du "dircom ». En effet, de plus en plus de compétences sont exigées de lui. Il reste garant des grandes orientations stratégiques du plan de communication, et maître dans l'utilisation des vecteurs et des techniques de communication interne. Mais il doit aussi, au quotidien, rassembler et exploiter toujours plus de données. Et surtout, effectuer une sélection systématique de celles-ci, en décidant de son propre chef et avec discernement de ce qui est utile - et à qui - de ce qui ne l'est pas.

Désormais « branché » en permanence sur des messageries internes ou externes, il reçoit en effet une masse de plus en plus importante d'informations, qu'il doit comprendre, analyser et synthétiser, remettre en forme et diffuser efficacement. Cela implique qu'il a aussi intégré, pour les utiliser, toutes les techniques propres aux nouveaux outils, qui exigent de lui une très haute technicité, et une remise à niveau constante. politique qu'il a à charge de mettre en œuvre. Désormais, il exerce une véritable ingénierie, maîtrisant des savoirs multiples, et des données qu'il doit exploiter en « flux tendu ", puis adapter à différents publics afin de les rendre opérationnelles pour ceux-ci. À lui de savoir s'adapter, pour être à la hauteur de ses nouvelles fonctions, et de contraintes techniques, sociales, politiques et budgétaires toujours plus drastiques et pressantes.

67 Mais ces messageries et ces réseaux induisent aussi de nouvelles attitudes pour les récepteurs, par rapport aux informations qu'ils font transiter. Les données ne sont plus distribuées prêtes à êtres lues et exploitées par ses «usagers", ce qui incitait à des comportements somme toute passifs. De plus en plus souvent, il faudra aller à cette information, en consultant régulièrement son ordinateur et sa boîte aux lettres électronique, en triant ce qui va être utile pour les tâches à accomplir, ou en fonction de ses centres d'intérêt. Et le plus difficile reste encore d'aller à l'essentiel, et de ne pas passer des heures à « papillonner » (d'aucuns diraient surfer).

À la logique du push, qui voyait les informations être poussées vers leurs utilisateurs, se superpose la logique du pull. Là, c'est cet utilisateur qui vient « tirer » avec éclectisme des «machines à communiquer» ce dont il va avoir besoin. Ou ce qui lui plaît, tout simplement. Cela signifie un certain effort, une démarche volontaire.

Il s'agit d'une révolution, une de plus. Celle-ci n'est pas seulement technologique, mais concerne les mentalités. Et les guerres les plus difficiles à gagner sont celles dont les combats se livrent dans les têtes... 


\section{Le journal interne, un projet de nature aussi humaniste}

70

si noir), la presse d'entreprise, entendue dans son acception noble, contribue à faire
l'apologie du lien; elle peut incarner une fonction de médiation essentielle au groupe, en ce qu'elle fait communiquer et participer, et déjà autour de son projet. Cette presse pratique en règle générale un journalisme salutaire: celui de la proximité, parfois affective, en tout cas géographique et d'intérêts. Fait rare, elle se nourrit et ne vit que des attentes, des préoccupations, des compétences, aussi, de ses lecteurs. Elle instaure convivialité et collégialité au sein des organisations, et pour cela, il faut lui rendre hommage, quels que soient les griefs dont on peut la trouver justiciable. Avant d'être un contenu, le journal interne est d'abord une relation inestimable, puisque dans l'idéal, elle œuvre à dépasser (ou du moins oublier un temps) les hiérarchies, les habitudes, les préjugés. Le journal d'entreprise est porteur d'un projet généreux: rapprocher les hommes et leur apprendre à mieux se connaître au sein d'une organisation, éclairer celleci d'une clarté finalement démocratique.

ettons en garde, une dernière fois, contre les tentatives d'aliénation ou de perversion desquelles ce grand dessein peut être la cible: censure, inféodation, langue de bois et propagande. Albert Camus, qui fut un grand journaliste avant d'être un grand écrivain, disait qu' « un pays vaut souvent ce que vaut sa presse. » Et il est vrai que cette liberté refusée ou concédée à la presse est le meilleur des baromètres démocratiques.

Le changement d'échelle, de la société à l'organisation, n'invalide pas l'aphorisme de Camus. Et c'est sans doute sur ce terrain de la démocratie que l'entreprise du troisième millénaire gagnera définitivement le statut qu'elle revendique parfois, «d'organisation citoyenne ». En donnant à chacun la liberté de s'informer librement, et de s'exprimer autrement que par la rumeur, la revendication, le silence ou la fuite. Le journal interne, dont les potentialités de développement sont encore grandes, devrait trouver là une mission renouvelée. Est-il utopique de prophétiser que la boucle de la production et du rendement comme finalités se bouclera un jour prochain? Au lieu de l'habituel profit économique, l'entreprise se tournera vers le profit des personnes, en se donnant pour mission de thésauriser son potentiel humain. La coïncidence est heureuse. Car l'information et la communication sont devenues des valeurs (aussi marchandes), et l'homme aura de plus en plus besoin d'elles pour se faire (re)connaître, en échangeant, en s'exprimant. La rencontre consacrée de l'homme et de la communication au sein des entreprises est capitale, autant qu'à capitaliser.

Puissent la logique et les structures capitalistiques ne pas obérer ce qui par-delà les modes et le pragmatisme, reste une utopie en marche. 


\section{BIBLIOGRAPHIE}

BEAU Dominique, DAUDEL Sylvain, Stratégie d'entreprise et communication, paris, Dunod, 1992.

CoRmerais Franck, Milon Alain, La Communication ouverte, Paris, Éditions Liaisons, 1994.

DonNET Georges, La Presse d'entreprise, mode d'emploi, Paris,Éditions Liaisons, 1994.

DUPUY Emmanuel, DeVERS Thomas, RAYNAUd Isabelle, La Communication interne, vers l'entreprise transparente, Paris,Les Éditions d'organisation, 1988.

FLORIS Bernard, La Communication managériale. La modernisation symbolique des entreprises, Grenoble, Presses Universitaires de Grenoble, 1996.

HenRIET Bruno, BONEu François, Audit de la communication interne, Paris, Les Éditions d'Organisation, 1990.

LARDELlier Pascal, Le Journal d'entreprise ; les ficelles du métier, Paris, éditions d'Organisation, 1998.

LE Goff Jean-Pierre, Le Mythe de l'entreprise, Paris, LaDécouverte, 192.

LEHNISCH Jean-Pierre, La Communication dans l'entreprise, Paris, Presses Universitaires de France, 1991.

LEKIME Fernand, La Presse d'entreprise, un phénomène médiatique du XXe siècle, Bruxelles, Éditions Labor, 1990.

MouillaUd Maurice, TETU Jean-François, Le Journal quotidien, Lyon, Presses Universitaires de Lyon, 1989.

WALTER Jacques, Les Directeurs de communication, Paris, L'Harmattan, 1995.

\section{NOTES}

1. Il serait faux de penser ici qu'une stricte bipartition oppose les journaux du secteur privé, à la facture professionnelle irréprochable, aux journaux de veine amateur du Service public. Rappelons qu'en 1995, CIVIC, le journal du Ministère de l'Intérieur, a obtenu le grand Prix des journaux d'entreprises, décerné annuellement par l'UJJEF, Union des Journaux et Journalistes d'entreprises de France. Dont acte.

2. Patrick Bachellerie, La Communication ouverte, de F. Cormerais et A Milon, Éditions Liaisons, 1994, p. 17.

3. Se reporter a La Communication managériale. La modernisation symbo-lique des entreprises. de Bernard Floris, Presses Universitaires de Grenoble, 1996.

4. Extrait des statuts de l'UJJEF.

5. Jean-Pierre Le Goff, dans le Mythe de l'entreprise (éditions LaDécouverte, 1992 à, se livre a une critique véhémente de ces chartes, qui tendent à faire accepter, en les légitimant, les valeurs entrepreneuriales Pourtant, chaque fois que nous avons mis en place un journal interne, nous avons proposé aux différents acteurs d'édicter et de cosigner une charte du journal interne, garante de quelques grands principes, tels que transparence, collégialité, respect, indépendance (relative)... Ces chartes ne sont en rien des Tables de la Loi, mais davantage des garde-fous, 
rappelant en cas d'égarement les valeurs fondatrices. Mais elles peuvent aussi jouer le rôle de fusible, sachant que la transgression par certains des protagonistes de ce qu'elle édictent implique forcément un recadrage, une mise à plat du projet.

6. Selon Taylor, d'ailleurs, deux flux principaux circulent dans l'entreprise : un flux d'énergie, qui transforme les matières premières, et un flux d'informations, celles-ci donnant les instructions que rendent cette transformation possible.

7. Rappelons que l'UJJEF est l'Union des Journalistes et Journaux d'Entreprises de France. Basée a Paris, cette association créée en 1947 œuvre a fédérer et a professionnaliser le secteur.

8. Source :Entreprisse, le journal de l'UJJEF. nº 65 Septembre 1995.

9. Notre ouvrage, Le Journal d'entreprise, les ficelles du métier, éditions d'Organisation, 1998, retrace un brève histoire de la presse d'entreprise, p. 201-205.

10. Précisons que le journal d'entreprise a connu, et connaît encore, d'autres supports que la papier. Son contenu peut ainsi se décliner sur des cassettes audio, vidéo, dans un réseau téléphonique, Minitel, et même télévisuel.

11. Rappelons qu'Intranet est constitué par un ensemble de réseaux "privés ", internes a des entreprises ou à des communautés, et auxquels n'accèdent que les personnes "autorisées ", munies des codes adéquats. Intranet est le réseau de prédilection pour les journaux internes. Quant a Extranet, il s'agit encore d'un réseau professionnel, mais connectant cette fois-ci des entreprises avec leurs clients, leurs fournisseurs et leurs sous-traitants.

12. Ainsi, Sert Schiesel, dans ne numéro 336 de Courrier international, daté du 10 avril 1997, nous apprend que certains sites Web liés a des journaux viennent de fermer, «en attendant une hypothétique rentabilité.» («Et deux de chute pour la presse en ligne!») Et nombreux sont les journaux à perdre quelques millions de dollars par an, en entretenant « pour l'image » un site qui rencontre bien moins d'adeptes que la « révolution numérique » pouvait en laisser supposer.

13. Selon le titre de l'ouvrage de Jacques Perriault, Flammarion, 1989.

\section{RÉSUMÉS}

La presse d'entreprise française traverse une période de bouleversements. Cette "crise de croissance » implique la nécessaire professionnalisation de ce secteur, autant qu'elle est liée à la réduction des budgets de communication; mais cette révolution est aussi technologique, eu égard à la migration actuelle de la presse d'entreprise sur des supports numériques. Se caractérisant par nombre de paradoxes et de malentendus, quant à ses missions et ses finalités, la presse d'entreprise peut-elle être saisie en tant que genre spécifique, et donner l'image d'une certaine cohérence, alors que l'analyse démontre qu'elle est hétérogène par nature ? Enfin, à quel projet autre que d'information le journal d'entreprise peut-il prétendre répondre? Ce texte s'efforcera de répondre à ces quelques questions.

The press of french enterprises is going throught upheaval states. This crisis first implies the necessary specifications of individuals for this particular field, but it is however relative to the budget cuts in the communication department; though, this revolution is mainly due to technology with respect to the actual migration to the press of enterprise now being on data processing. Those paradoxes and misunderstandings concern the missions and the finalities of this type of press. Does the press of enterprises can be understood as a specific type of press, and give the illusion of a certain coherence? Hence, the analysis shows otherwise that she is 
hetegeneous by nature. At last, to what kind of mission others than offering informations should the newsletter of enterprise can actually do for organizations? This paper will try to respond to some of these questions.

\section{AUTEUR}

\section{PASCAL LARDELLIER}

Pascal Lardellier est Maître de conférences en Communication à l'université de NiceSophia-Antipolis. Directeur d'une maîtrise Art et développement culturel, il vient de publier Le Journal d'entreprise, aux éditions d'Organisation, et de diriger le numéro 92-93 de la revue internationale de sémiologie Degrés, dont le thème est « Penser le multimédia ». 